\title{
Weather regime dependence of extreme value statistics for summer temperature and precipitation
}

\author{
P. Yiou ${ }^{1}$, K. Goubanova ${ }^{2}$, Z. X. Li ${ }^{2}$, and M. Nogaj ${ }^{3}$ \\ ${ }^{1}$ Laboratoire des Sciences du Climat et de l'Environnement/IPSL, CE Saclay l'Orme des Merisiers, 91191 Gif-sur-Yvette, \\ France \\ ${ }^{2}$ Laboratoire de Météorologie Dynamique/IPSL, 4 Place Jussieu, 75005 Paris, France \\ ${ }^{3}$ EDF, Département Mécanique des Fluides, Energies et Environnement, 78401 Chatou, France
}

Received: 17 September 2007 - Revised: 4 April 2008 - Accepted: 4 April 2008 - Published: 6 May 2008

\begin{abstract}
Extreme Value Theory (EVT) is a useful tool to describe the statistical properties of extreme events. Its underlying assumptions include some form of temporal stationarity in the data. Previous studies have been able to treat long-term trends in datasets, to obtain the time dependence of EVT parameters in a parametric form. Since there is also a dependence of surface temperature and precipitation to weather patterns obtained from pressure data, we determine the EVT parameters of those meteorological variables over France conditional to the occurrence of North Atlantic weather patterns in the summer. We use a clustering algorithm on geopotential height data over the North Atlantic to obtain those patterns. This approach refines the straightforward application of EVT on climate data by allowing us to assess the role of atmospheric variability on temperature and precipitation extreme parameters. This study also investigates the statistical robustness of this relation. Our results show how weather regimes can modulate the different behavior of mean climate variables and their extremes. Such a modulation can be very different for the mean and extreme precipitation.
\end{abstract}

\section{Introduction}

The impact of the atmospheric circulation on the temperature and precipitation conditions in the extra-tropics has been documented in many studies (e.g. Hurrell et al., 2003, for a review). Such a relation is well documented for the last

Correspondence to: P. Yiou

(pascal.yiou@cea.fr)
50 years. It has also been argued that reorganizations of the atmospheric circulation, related to natural variability or caused by a forcing, can cause changes in midlatitude surface temperature (Corti et al., 1999; Kageyama et al., 1999; Palmer, 1999). This relation is also a basis for climate proxy reconstructions (Bradley, 1999; Luterbacher et al., 1999) of mean atmospheric flow or temperature. In many cases, ecosystems are more sensitive to climate extremes than to its mean variations. This calls for an evaluation of this relation for temperature and precipitation extremes.

In this paper, we use the paradigm of weather regimes to describe quasi-stationary states of the atmosphere (Cheng and Wallace, 1993; Ghil and Robertson, 2002; Michelangeli et al., 1995; Vautard and Legras, 1988; Vautard et al., 1988; Vautard, 1990). Weather regimes emerge from a statistical description of the atmosphere into discrete sets and are generally obtained from statistical classification or estimates of a high dimensional probability density function. Hence, atmospheric circulation can be viewed as a Markov process, with state transitions with probability distributions to be evaluated (Ghil and Robertson, 2002). The relation between weather regimes and local extreme events of temperature or precipitation has been investigated by a few authors (Cassou et al., 2005; Plaut et al., 2001; Plaut and Simonnet, 2001; Robertson and Ghil, 1999; Yiou and Nogaj, 2004; Yiou et al., 2006). In those studies, extreme events are defined as exceedances of a fixed threshold, but in general the probability distribution of the extremes is not used to build the relation between the circulation and surface extremes.

Extreme Value Theory (EVT) is a useful paradigm to quantify the statistical properties of extreme events (Coles, 2001). EVT aims at describing the tail of the distribution

Published by Copernicus Publications on behalf of the European Geosciences Union and the American Geophysical Union. 
Table 1. Table of acronyms and abbreviations.

\begin{tabular}{ll}
\hline Acronym & Definition \\
\hline BLO & Blocking \\
ECA & European Climate Assessment \\
EOF & Empirical Orthogonal Function \\
EVT & Extreme Value Theory \\
GA & Greenland Anticyclone \\
GPD & Generalized Pareto Distribution \\
IID & Independent and Identically Distributed \\
JJA & June-July-August \\
NAO & North Atlantic Oscillation \\
NCEP & National Centers for Environmental Prediction \\
PC & Principal Component \\
POT & Peak-Over-Threshold \\
RL & Return Level \\
RR & Daily precipitation \\
TG & Daily mean temperature \\
TX & Daily maximum temperature \\
z500 & Geopotential height at 500 hPa \\
\hline
\end{tabular}

of random variables, and stems from a convergence theorem stating that the distributions of extremes follow generic laws, provided a few elementary hypotheses are verified (Leadbetter et al., 1982). EVT supersedes a Gaussian framework when extreme values are treated and allows for much finer data descriptions (Cooley et al., 2006; Naveau et al., 2005). Hence we place this paper in the EVT framework to describe extremes of temperature and precipitation, which as will be shown, do not follow Gaussian distributions. This paper investigates the relation between the EVT parameters of variables (temperature and precipitation) and a controlling variable acting like a Markov chain (atmospheric circulation). The classification method, a stabilization procedure, and EVT theory are exposed in Sect. 2. The data sets are described in Sect. 3. Results of the analyses on temperature and precipitation in France, including statistical tests, are given in Sect. 4. Conclusions and perspectives appear in Sect. 5. The acronyms and abbreviations used in the text are listed in Table 1.

\section{Methodology}

\subsection{Weather regimes and classification}

It has been shown that surface temperature and precipitation conditions depend on patterns of the atmospheric circulation in the northern extra-tropics (Corti et al., 1999; Palmer, 1999; Robertson and Ghil, 1999). Moreover it has been argued that recent decadal changes in mean temperature can be caused by circulation reorganizations only (Corti et al., 1999; Palmer, 1999).
The hemispheric atmospheric circulation varies on time scales of days. Many arguments have been proposed to project this variability in the Northern Hemisphere onto a small number of quasi-stationary states (Cheng and Wallace, 1993; Ghil and Robertson, 2002; Vautard and Legras, 1988; Vautard, 1990). Such states are called weather regimes and provide a discrete description of the atmospheric circulation. The determination of those states is a statistical problem of classification: the weather regimes are the centroids of a cloud of points in an ad hoc phase space (Cheng and Wallace, 1993; Michelangeli et al., 1995). The attribution of each atmospheric circulation observation to a centroid defines the clusters of the classification.

A classical way of performing this classification is to use a k-means algorithm (Hartigan and Wong, 1979). This straightforward classification method partitions a dataset into a predefined set of $k$ clusters, such as to minimize the spread within them. The algorithm then iteratively searches for the best partition, given random initial centroids. Hence, the resulting classification is a priori random. Repeating this classification procedure with random initializations and checking for the reproducibility of the centroids can provide a heuristic criterion to assess the validity of the partitioning into distinct $k$ clusters (Michelangeli et al., 1995).

Other classification methods have been used on atmospheric circulation data (Beck et al., 2007; Corti et al., 1999; Smyth et al., 1999; Vrac et al., 2007), but results are generally consistent from one method to another. The advantage of the k-means algorithm is that its straightforward application is computationally efficient.

Atmospheric circulation datasets over the North Atlantic region generally contain $\approx 10^{3}$ grid points on a daily time scale (see Sect. 3.1). In order to maintain a convergence of the classification algorithms, it is necessary to highly reduce the number of variables. This is done with a principal component (PC) analysis (von Storch and Zwiers, 2001) and keeping the first $P$ modes that represent, for instance, more than $90 \%$ of the variance. Thus the corresponding empirical orthogonal functions (EOFs) contain the reduced phase space on which the classifications are performed.

Winter atmospheric circulation around the North Atlantic is generally classified into four weather regimes (Corti et al., 1999; Kimoto and Ghil, 1993a,b; Michelangeli et al., 1995), regardless of the method used to determine the classification. This is due to the large daily variability of the circulation, which yields important swings that are physically and statistically identifiable. In the summer, the circulation of the North Atlantic has less variability and the classification of atmospheric circulation data is generally less clear, if not invalid. In this paper we do not attempt to prove that the summer circulation is classifiable or to find the "best" number of clusters that describes it. Visual inspections of projections of atmospheric circulation data onto two of the main PCs do not suggest that there exist clearcut clusters. Thus the criterion devised by Michelangeli et al. (1995) would reject the 
data classifiability for summer atmospheric circulation. Our choice is rather to "cut the cake" into a small number of parts (for instance $k=4$ regimes) which make statistical sense in a way defined below.

Our strategy is to perform a large number (typically, $n=100)$ of k-means classifications of the data with different initializations and the same number $(k=4)$ of clusters. Then we determine the most probable set of centroids found by the classifications. To do this, we classify the set of $k n(=400)$ clusters with a mixture modelling algorithm that determines an optimal number of centroids (Smyth et al., 1999; Vrac et al., 2007). Hence we obtain a set of $k^{\prime} \geq 4$ meta-clusters for the $k n$ clusters obtained by k-means. The most frequent meta-cluster then is determined. We then compute a composite centroid from the population of this most frequent metacluster. This multi-step procedure ensures that the obtained $\mathrm{k}$-means classification is stable in a quantifiable sense, although it is acknowledged that the data are not necessarily classifiable. We conjecture that this procedure takes into account local irregularities of the data outer boundaries but this discussion is beyond the scope of this paper. An alternative method was proposed to define modes of variability of continuously varying data (Bernacchia and Naveau, 2007). We stress that the key to this stabilization procedure is that the number of clusters has to be specified in advance. For the summer data, $k=4$ clusters are specified for the North Atlantic atmospheric circulation, which is physically reasonable (Cassou et al., 2005).

In this study, we consider a classification of the atmospheric circulation which is by no means unique, but which is the most probable with a k-means algorithm. The clusters of this classification are the so-called weather regimes.

\subsection{EVT and POT}

Analysis of the distribution of extremes is an important diagnostic tool for investigating the occurrence of rare events (Gumbel, 1958; Coles, 2001; Leadbetter et al., 1982; Naveau et al., 2005) This statistical approach was pioneered by Gumbel (1958), and has proved to be efficient in the fields of finance (Embrechts et al., 1997), hydrology (Katz, 1999; Katz et al., 2002; Yiou et al., 2006) and climatology (Kharin and Zwiers, 2000; Naveau and Moncrieff, 2003; Nogaj et al., 2006). The general idea of the Extreme Value theory (EVT) is to parameterize the tail of the distribution of climate variables, which contains information about the distribution of extremes (Gumbel, 1958). For instance, a Gaussian distribution has a thin tail with a "low" probability of observing large events, whereas a distribution with a heavy tail yields a relatively high probability of having large values and has infinite higher moments. We summarize hereafter the general properties of extreme values.

The Peak-Over-Threshold (POT) method describes the probability density function of a variable when it exceeds a high threshold. Considering an independent identically dis- tributed (IID) random variable $X$, of continuous distribution $F$, a given fixed threshold $u$ and any number $x$ the conditional probability $F_{u}(x)$ that $X$ exceeds $x$, given that $X$ exceeds $u$ is:

$F_{u}(x)=\mathbf{P}(X>x \mid X>u)=\frac{1-F(x)}{1-F(u)}$, for $x>u$.

The aim of the POT method is to describe $F_{u}(x)$ independently of the a priori unknown $F$. Under conditions of convergence and proper normalization (Embrechts et al., 1997), and for large enough $u$, this distribution can be approximated by the generalized Pareto distribution (GPD) with parameters $\sigma$ (scale parameter) and $\xi$ (shape parameter):

$H(x)=1-\left(1+\frac{\xi(x-u)}{\sigma}\right)^{-1 / \xi}$, for $x>u$

defined for $\xi \neq 0$. If $\xi=0$, the GPD estimate becomes an exponential distribution:

$H(x)=1-\exp \left(-\frac{x-u}{\sigma}\right)$, for $x>u$.

Those parameters relate closely to the usual extreme value parameters that describe the distribution of maxima of $X$ (Coles, 2001). In this paper, we estimate the GPD parameters by a classical maximum likelihood method (Coles, 2001). Confidence intervals obtained from this procedure are used to assess the significance of parameter estimates. The choice of the threshold $u$ is a compromise between the convergence of Eqs. (2), (3) toward a GPD distribution, and the number of data to estimate the GPD parameters. Heuristic (and necessary arbitrary) procedures can be devised for this choice (Coles, 2001). Here we use as threshold $u$ the upper 90th percentile of the data, implying that the parameters in Eqs. (2), (3) are estimated with $10 \%$ of the data. We verified that taking the 95th percentile does not significantly change our results.

The main caveat of this approach is that the conditions of convergence toward a GPD distribution are not necessarily met, because this mathematical result is asymptotic for large datasets and large threshold $u$. It is possible to check the goodness of fit of the model of Eqs. (2), (3) with classical quantile-quantile (Q-Q) plots (Coles, 2001). Hence we checked that the Q-Q plots of time series we analyzed are significant within $95 \%$ confidence intervals.

The climate variables, daily temperature and precipitation, are generally correlated from one day to the next and are not independent. Hence the POT method cannot be applied in a straightforward way to such data (Coles, 2001; Nogaj et al., 2006). For a given variable $X$, we thus determine clusters of exceedances of $X$ above a threshold $u$. The maxima of each cluster can be considered independent (and identically distributed). Hence we apply the POT method on declustered data. In this study we assume that clusters of exceedances are separated by at least one day under the threshold. Other, 
more sophisticated definitions for clusters can be used, but we chose the simplest one for illustration purposes. An extremal index $\theta$ can be defined to measure the short-term dependence of data. This index is a normalizing exponent for which the distribution of extremes of the dependent data converges to a GPD distribution (Coles, 2001). It can be estimated by

$\hat{\theta}=\frac{n_{c}}{n_{u}}$,

where $n_{c}$ is the number of clusters and $n_{u}$ is the number of exceedances of the threshold $u$. The parameter $\theta$ is equal to 1 when the data are independent and decreases with the typical size of clusters.

In this study, we assume for simplicity that the processes and their extremes are stationary, although trends have been identified in temperature extremes (Nogaj et al., 2006) in Europe. High summer temperatures in France do not seem to yield such trends in GPD parameters (Nogaj et al., 2006), therefore this technical hypothesis is reasonable if we restrict our analysis to meteorological data in France (see Sect. 3).

Rather than looking only at the scale and shape parameters of the GPD distributions, it is sometimes more practical to compute return levels associated with a return period. We define a return level (RL) $z_{T}$ corresponding to a return period of $T$ years by the expectation of the event "to exceed the RL" to be equal to one during that period (Yiou et al., 2006). For an IID process, the RL is determined from the inversion of Eqs. (2), (3):

$z_{T}=u+\frac{\sigma}{\xi}\left[\left(T n_{X} \zeta_{u}\right)^{\xi}-1\right]$, for $\xi \neq 0$,

and

$z_{T}=u+\sigma \log \left(T n_{X} \zeta_{u}\right)$, for $\xi=0$,

where $n_{X}$ is the number of observations of $X$ per year $\left(N=T n_{X}\right.$ is the total number of observations) and $\zeta_{u}$ is the probability of exceeding the threshold $u$ (Coles, 2001). If seasonal extrema are considered, for instance summer extreme hot temperatures, $n_{X}$ is set to 92 , the number of days from June to August. The probability $\zeta_{u}$ is estimated by $\hat{\zeta}_{u}=\frac{n_{u}}{N}, n_{u}$ being the number of exceedances of $u$. The nonstationary case, i.e. with time varying GPD parameters, is much more complicated to deal with (Nogaj et al., 2006). If the data is dependent and clusters of extremes are found, the RL estimate $z_{T}$ has to be rectified to:

$z_{T}=u+\frac{\sigma}{\xi}\left[\left(\operatorname{Tn}_{X} \zeta_{u} \theta\right)^{\xi}-1\right]$, for $\xi \neq 0$,

because the EVT is applied on the cluster maxima instead of the whole dataset.

The variations of RL $z_{T}$ as a function of the return period $T$ depend on the shape parameter $\xi$ which controls the convexity of this relation Eq. (5). The relation of Eqs. (5), (6) can be used to extrapolate RLs to return periods that are longer than the period of observation. This illustrates the predictive virtue of EVT, although it should be noted that confidence intervals grow rapidly with the return period $T$. In the following, we will compute RLs associated with return periods of 50 years, i.e. levels exceeded on average once during the observation period.

\subsection{Imposing regime dependence}

With the hypothesis that weather regimes represent different climatic states affecting mean temperature and precipitation, we investigate the extreme properties during weather regimes. Thus we determine the probability of the variable $X$ exceeding a value $x$, given that $X$ is larger than a threshold $u$ and an exogenous variable $Y$ (i.e. the weather regime to which belongs the daily atmospheric circulation) is in a state $k$. If the marginal probability density function of $X$ when $Y$ is in state $k$ is denoted $F^{(k)}(x)$, we want to estimate:

$$
\begin{aligned}
F_{u}^{(k)} & =\mathbf{P}\left(X>u \mid X>u^{(k)}, Y=k\right) \\
& =\frac{1-F^{(k)}(x)}{1-F^{(k)}\left(u_{k}\right)}, \text { for } x>u^{(k)} .
\end{aligned}
$$

In Eq. (8), $u^{(k)}$ is a state-dependent threshold, which could be set to a high value independent of $Y$. Under IID hypotheses on $X$, we can hence determine GPD parameters $\sigma^{(k)}$ and $\xi^{(k)}$ for each state $k$. Confidence intervals are obtained in the same fashion as for the classical case.

Return levels of extremes of $X$ can be estimated by a slight modification of Eq. (7) taking into account $\pi^{(k)}$, the mean frequency of weather regime $k$, which is its probability of occurrence. We obtain:

$$
\begin{aligned}
z_{T}^{(k)}= & u^{(k)}+\frac{\sigma^{(k)}}{\xi^{(k)}}\left[\left(\operatorname{Tn}_{X} \zeta_{u^{(k)}} \theta^{(k)}\right)^{\xi^{(k)}}-1\right], \\
& \text { for } \xi^{(k)} \neq 0,
\end{aligned}
$$

where $\theta^{(k)}$ and $\zeta_{u^{(k)}}$ are respectively the extremal index for regime $k$ and probability of exceeding the threshold $u^{(k)}$. The $\mathrm{RL}$ equation for $\xi^{(k)}=0$ is obtained from Eq. (6).

It is useful to test the significance of the dependence of the parameters $u^{(k)}, \sigma^{(k)}$ and $\xi^{(k)}$ to the regime $k$, i.e. their difference with the parameters $u, \sigma$ and $\xi$ obtained from Eq. (2) without taking weather regimes into account. To this end, the null hypothesis of no weather regime dependence is introduced and tested. Hence we determine the probability density function of each GPD parameter, given a random regime distribution. This is performed by bootstrap simulations of the regime attribution $Y$ (Efron and Tibshirani, 1993). This approach circumvents the problem that the probability distribution of the GPD parameters is unknown, and has to be estimated empirically. Here, $S=100$ random shuffles of the daily weather regime attribution are performed, in order to erase the potential weather regime dependence of temperature or precipitation extremes. This procedure preserves the 
frequencies $\pi^{(k)}$. The parameters $u^{(k)}, \sigma^{(k)}$ and $\xi^{(k)}$ are estimated for each bootstrap simulation. From this set of experiments, we can estimate the probability distribution of the parameters with a null hypothesis of no weather regime dependence. The null hypothesis can be rejected when the weather regime dependent GPD parameters (Eq. 8) are outside of chosen quantiles of the bootstrap experiments (e.g. the 1st and 99th quantiles, or other quantiles).

A convenient way of visualizing the distribution of the GPD parameters with the bootstrap experiments is to use box-and-whisker plots featuring quantiles of the empirical probability distributions. The box-and-whisker plots we use in this paper feature the 25th, 50th and 75th quantiles of the distribution of estimated parameters (resp. $q_{25}, q_{50}$ and $q_{75}$ ). Such plots also represent the values of $q_{25}-1.5 \times\left(q_{75}-q_{25}\right)$ and $q_{75}+1.5 \times\left(q_{75}-q_{25}\right)$, which are proxies for extremes. Since the distribution of the parameters $u^{(k)}, \sigma^{(k)}$ and $\xi^{(k)}$ are approximately Gaussian (Embrechts et al., 1997), the two latter values encompass the 0.5 and 99.5 th quantile. Therefore, if the regime dependent estimate of a parameter falls outside those "whiskers", it can be considered significantly different from the "non dependent" estimate.

This approach of weather regime dependence is similar to the mixture model of Vrac et al. (2007) who used a Bayesian model for precipitation taking into account weather typing. Our study focuses on the extremes and tries to assess their weather regime dependence.

\section{Data}

\subsection{Atmospheric circulation}

The weather regimes were determined from the National Centers for Environmental Prediction (NCEP) reanalysis data between 1948 and 2007 (Kalnay et al., 1996). We used the daily mean geopotential height at $500 \mathrm{hPa}$ (z500) during the summer season (June-July-August, JJA). The horizontal resolution of z500 is $2.5^{\circ}$ by $2.5^{\circ}$ in longitude and latitude.

We removed the seasonal cycle by simply computing daily averages for each day of the year over the whole period and for each gridpoint. The obtained seasonal cycle is generally irregular and we smooth it with a cubic spline smoothing algorithm (Green and Silverman, 1994; Hastie and Tibshirani, 1990). We hence treat anomalies of the atmospheric circulation with respect to an idealized seasonal cycle.

The atmospheric patterns that control European climate generally cover the North Atlantic region. Thus, we focused on the region between $80^{\circ} \mathrm{W}$ and $50^{\circ} \mathrm{E}$ in longitude, and $25^{\circ} \mathrm{N}$ and $70^{\circ} \mathrm{N}$ in latitude. Since the surface of grid cells depends on latitude, the z500 data was normalized by the cosine of latitude. We kept 14 PCs of z500, hence capturing $90 \%$ of the total variance. We imposed a number of weather regimes for the JJA data, to be consistent with Cassou et al. (2005); Vautard et al. (2007).

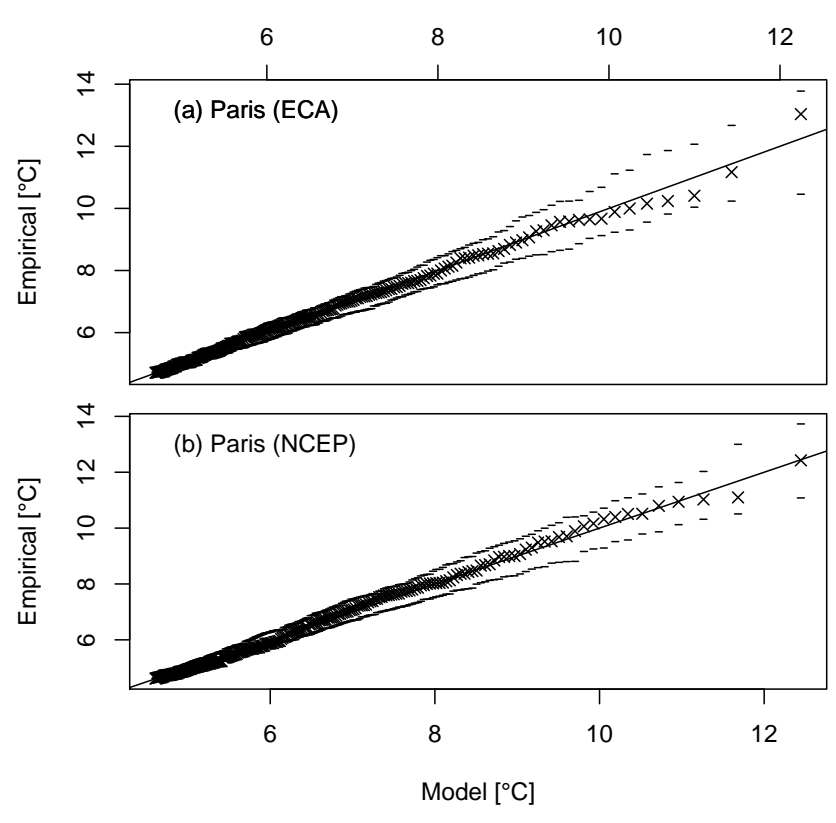

Fig. 1. Quantile-quantile (Q-Q) plots for the Generalized Pareto Distribution (GPD) model of anomalies of summer (June to August) mean daily temperature (TG) in Paris from the ECA data (KleinTank et al., 2002) (panel a) and the closest gridpoint from the NCEP reanalysis (Kalnay et al., 1996) (panel b). Crosses (x) indicate data points. $95 \%$ confidence intervals are indicated in dashed lines.

\subsection{Temperature and precipitation}

We used the European Climate Assessment (ECA) dataset (Klein-Tank et al., 2002) for temperature and precipitation. We took daily maximum temperature (TX), mean temperature (TG) and daily cumulative precipitation (RR) for station data. Many datasets of the ECA database start before 1900 . In this study, we focus on stations in France because it was shown earlier (Nogaj et al., 2006) that the GPD parameters of temperatures do not have a trend, and hence the EVT can be applied in a simple stationary framework.

We filtered out stations with more than a total of five years of missing data before 1948. Hence we retained 11 stations for RR and 27 stations for TX (and TG).

The use of observed temperature or precipitation rather than reanalysis data is made necessary because NCEP reanalysis precipitation extremes seem to be biased toward thin tailed distributions. We illustrate this by looking at the Paris data as an example. We isolate the closest gridpoint to Paris of the NCEP reanalysis and compute the daily JJA total. Note that the closest gridpoint is not the same for temperature and precipitation, because the grids are not the same for those two variables. In this first illustration, we used the TG series rather than TX because the NCEP reanalysis does not simulate TX, but has a 6-hourly or daily resolution. Hence for this comparison exercise, it is preferable to use variables that 

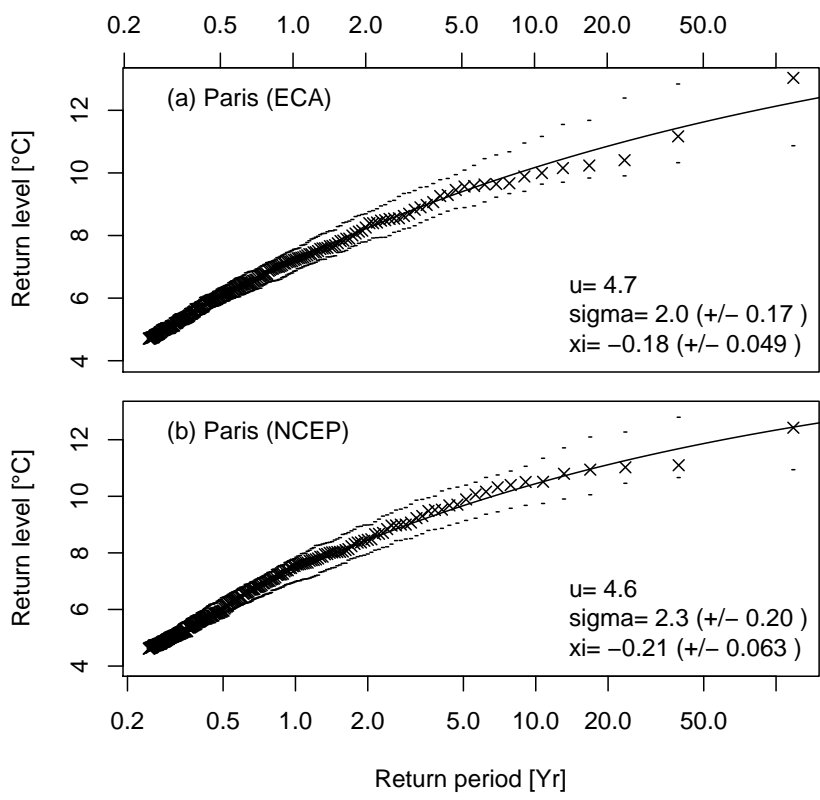

Fig. 2. Return levels for anomalies of summer (June to August) mean daily temperature (TG) in Paris from the ECA data (KleinTank et al., 2002) between 1948 and 2007 (panel a) and the closest gridpoint from the NCEP reanalysis (Kalnay et al., 1996) (panel b). Return periods are expressed in years, and return levels in ${ }^{\circ} \mathrm{C}$. Crosses $(x)$ indicate data points. For comparison purposes, horizontal and vertical axes have the same range for both panels. Generalized Pareto Distribution (GPD) parameters (threshold $u$, scale $\sigma$ and shape $\xi$ ) are indicated in each panel. 95\% confidence intervals are indicated in dashed lines.

have similar meanings, namely the mean daily temperature. We removed the seasonal cycle in both temperature series and treated anomalies with respect to this cycle.

The GPD parameters for the ECA (TG) and NCEP temperature and precipitation time series between 1948 and 2007 are then computed. The temperature data for the two datasets have a high linear correlation $\left(r=0.92\right.$, $\mathrm{p}$-value $\left.<10^{-5}\right)$. Thus it can be considered that the NCEP temperature data provides a faithful representation of daily variability. As expected, the GPD parameters are very close between the two time series, with a consistently negative shape parameter, indicating a finite tailed distribution. A comparison with other stations gives similar results for temperature (not shown). The QQ plots for the ECA TG and NCEP reanalysis indicate that the temperature anomaly extremes are well represented by the POT model within ad hoc confidence intervals (Fig. 1) The return level plots of the two series are shown in Fig. 2, indicating the same type of variability. This suggests that the NCEP reanalysis provides a good representation of temperature extremes. A systematic "proof" involving the comparison between temperature minima, and other seasons is beyond the scope of the paper.

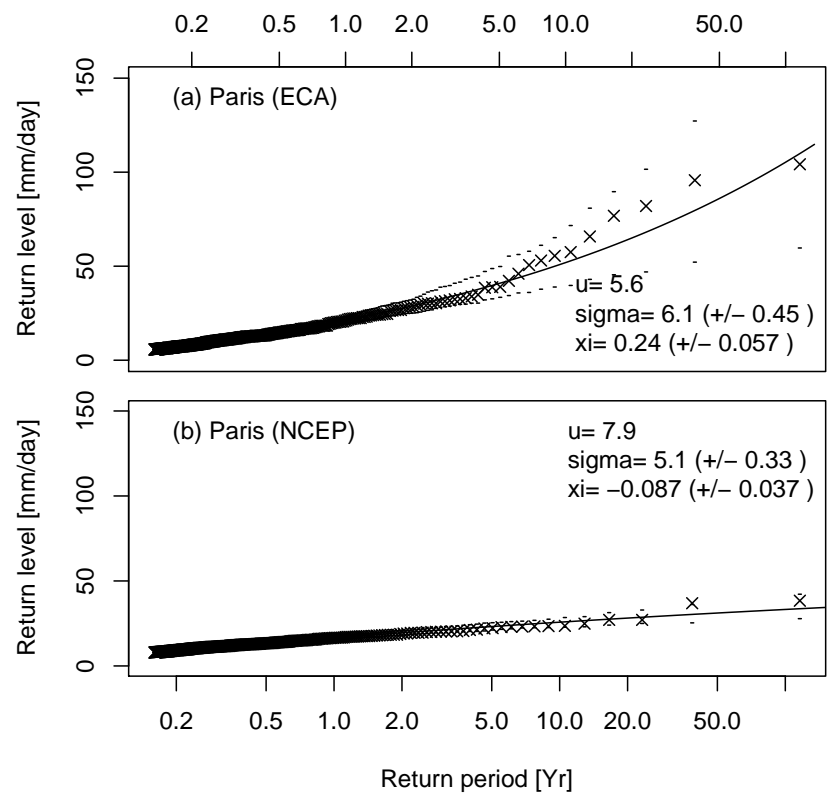

Fig. 3. Return levels for summer (June to August) precipitation (RR) in Paris from the ECA data (Klein-Tank et al., 2002) between 1948 and 2007 (panel a) and the closest gridpoint from the NCEP reanalysis (Kalnay et al., 1996) (panel b). Return periods are expressed in years, and return levels in $\mathrm{mm} /$ day. Crosses $(\times)$ indicate data points. For comparison purposes, horizontal and vertical axes have the same range for both panels. Generalized Pareto Distribution (GPD) parameters (threshold $u$, scale $\sigma$ and shape $\xi$ ) are indicated in each panel. Data points are indicated by crosses. $95 \%$ confidence intervals are indicated in dashed lines.

For the precipitation (RR) time series between 1948 and 2007, the shape parameter of the ECA Paris data is significantly positive, indicating a heavy tail, while the same parameter for the NCEP data is undistinguishable from 0 , i.e. an exponential distribution. For illustration purposes, the return level plots for RR are shown in Fig. 3. This figure shows that reanalysis precipitation data tend to be "normalized", which can be acceptable for mean values, but not for the study of extremes. Hence, it is unlikely that "extreme" precipitation events can be correctly estimated with NCEP data.

In order to illustrate the role of weather regimes on seasonal climate variations, we determined the ten warmest European summers by the procedure developed by Vautard et al. (2007) on TX data. This procedure takes the average European temperature for the summer (JJA) and determines the ten hottest years. The use of European stations (rather than French stations only) is motivated by the fact that European summer heatwaves generally develop in southern France and reach a climax in eastern France and central Western Europe. Since this area is not well covered in the ECA database, we prefer to extend the definition of European heatwaves. We used the same procedure to select the ten coldest and ten 

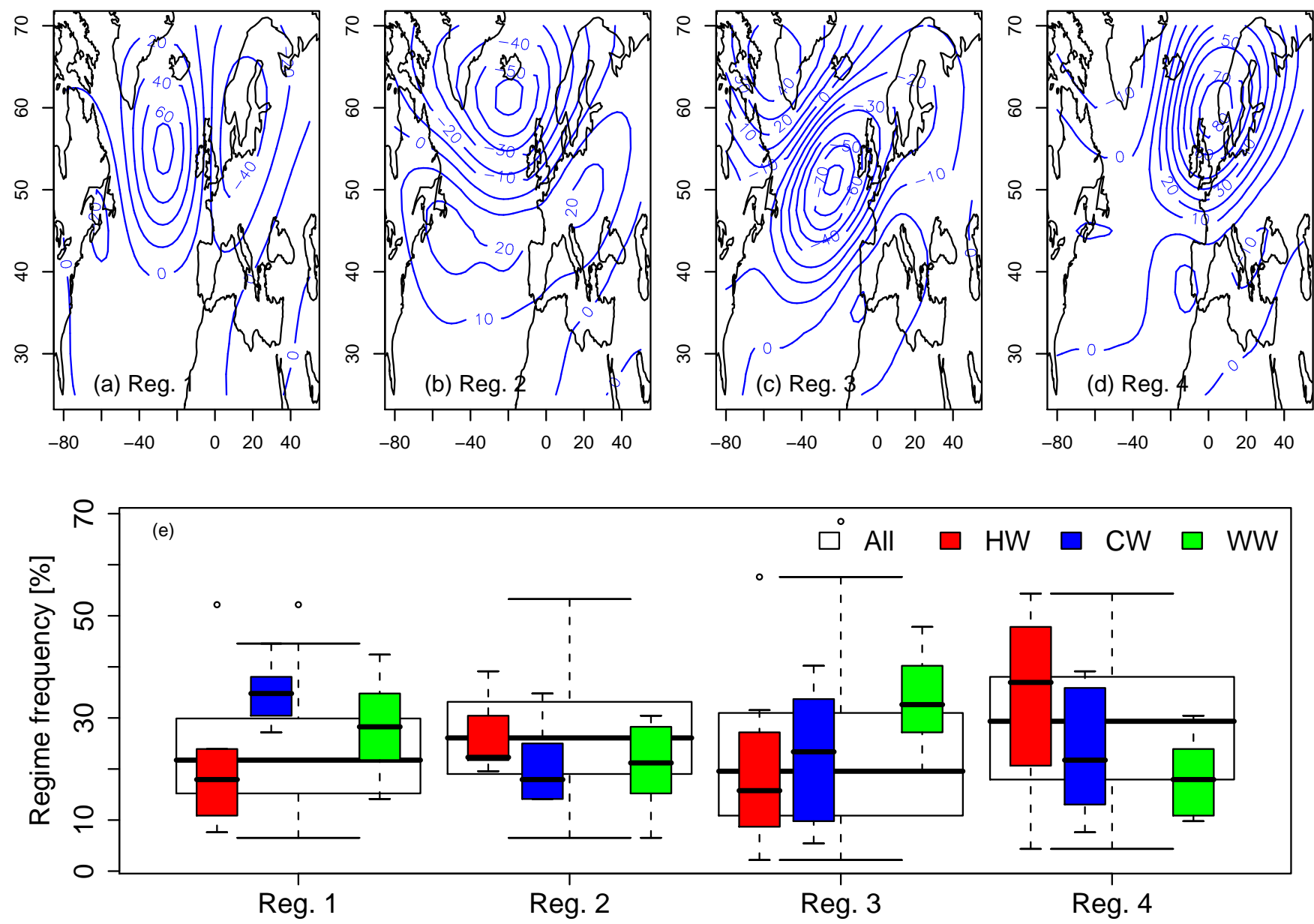

Fig. 4. Top panels (a-d): four weather regimes obtained with a k-means classification of daily summer geopotential height at $500 \mathrm{mb}$ (z500) of the NCEP reanalysis (Kalnay et al., 1996) between 1948 and 2007. Bottom panel (e): box and whisker plots of the frequency of each regime ("All", wide boxes). The thin colored boxes indicate the regime frequencies during the 10 hottest summers ("HW", red), 10 coolest summers ("CW", blue) and 10 wettest summers ("WW", green).

wettest summers in Europe from the TX and RR data, respectively.

It is a priori not obvious whether the warmest summers on average yield the highest daily temperatures, or whether the wettest summers have the highest daily precipitation. Our paper aims at clarifying this connection.

\section{Results}

\subsection{Classification}

The classification procedure explained in Sect. 2.1 was applied to z500 summer anomalies over the North Atlantic. The four weather regimes are shown in Fig. $4 a-d$. The first regime is reminiscent of the negative phase of the North Atlantic Oscillation (NAO-) and contains a strong positive z500 anomaly over the North Atlantic, with a negative anomaly over northern Europe. The second regime is the positive phase of the NAO (NAO+) and yields positive z500 anomalies extending from the US East coast to Western Europe. The third regime (Greenland Anticyclone, GA) shows a positive anomaly over Greenland and negative anomalies over the central North Atlantic. The fourth regime (Blocking, BLO) shows a strong positive anomaly over Scandinavia. Regimes 2 and 4 yield an anticyclonic circulation over western Europe, while regimes 1 and 3 favor cyclonic circulations. The four summer weather regimes differ from the ones of Cassou et al. (2005) because we use more PCs and our criterion to determine the most probable classification is not the same. However, we find consistent NAO phases and the blocking regimes.

The frequencies of those four weather regimes are shown in Fig. $4 \mathrm{e}$ (wide boxplots). The fat box and whisker plots indicate the 10,25, 50,75 and 90th quantiles of the seasonal 

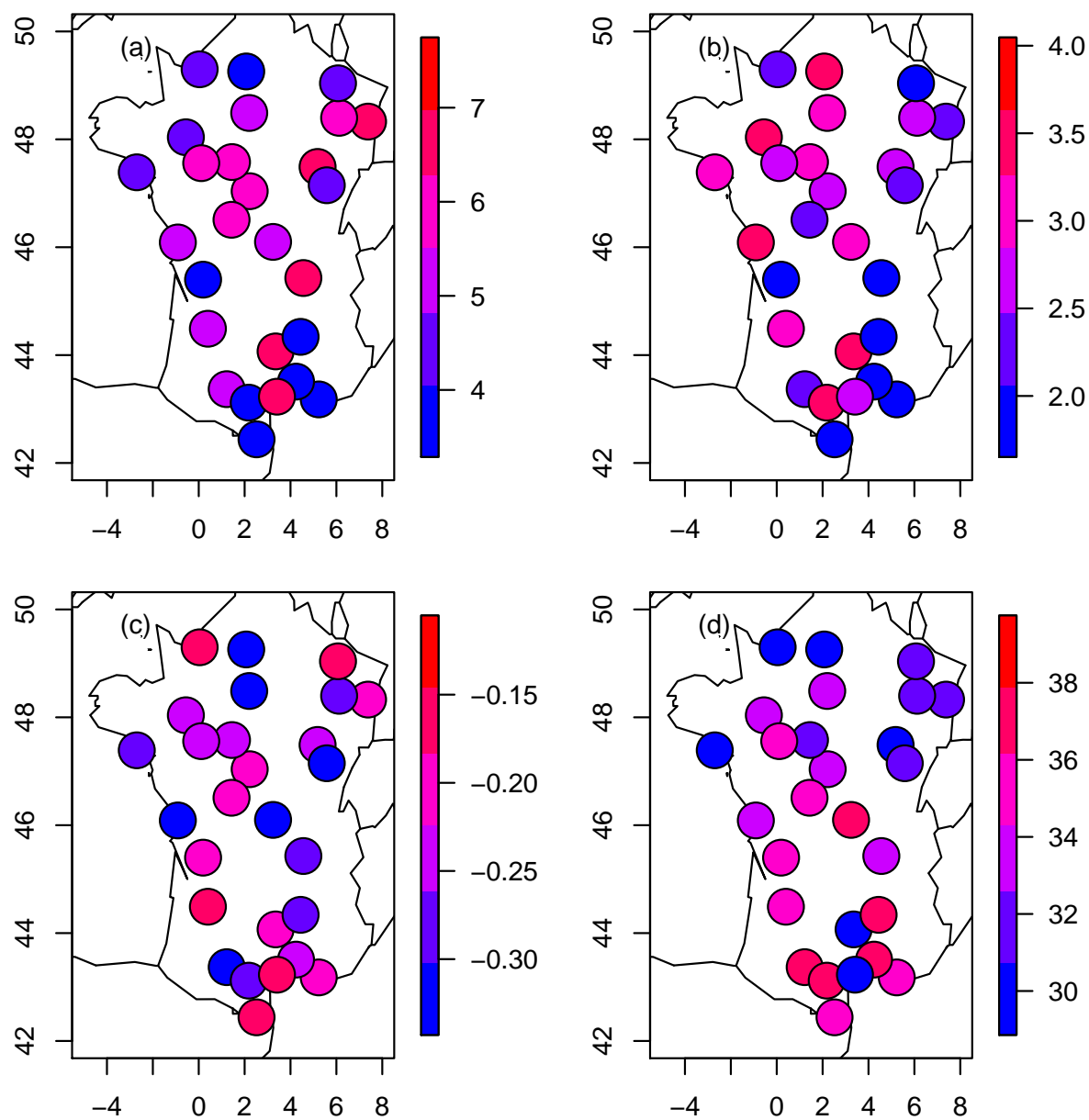

Fig. 5. GPD parameters of daily maximum summer temperature (TX) anomalies for the French ECA stations (Klein-Tank et al., 2002) between 1948 and 2007. Panel (a): threshold $u$ in ${ }^{\circ} \mathrm{C}$; (b) scale $\sigma$ in ${ }^{\circ} \mathrm{C}$; (c) shape $\xi$; (d) return level for 50 year period $\mathrm{RL}_{50}$ in ${ }^{\circ} \mathrm{C}$ added to the mean summer value.

regime frequency distribution. Overall, regimes $2(\mathrm{NAO}+)$ and 4 (BLO) are the most frequent on average (higher medians), but there is a high interannual variability in the regime frequency.

From the ECA temperature and precipitation dataset, we picked the ten hottest, coldest and wettest summers over summer. The weather regime frequencies were determined for those three types of extreme summers. They are denoted in Fig. 4e by red, blue and green boxes, respectively, and corresponding box and whisker plots. We hence illustrate that cold and wet summers are generally associated with cyclonic weather regimes (Regimes 1 and 3), and hot summers are associated with anti-cyclonic regimes, especially the Blocking regime 4 (Cassou et al., 2005). We find that extreme summers experience all the weather regimes, but their relative frequencies bear a signature of the type of extreme.

\subsection{EVT and its dependence on weather regimes}

We performed POT analyses on the TX seasonal anomalies and RR for the French stations. The threshold parameters were taken as the 90th quantile of each data set. The GPD parameters of TX summer anomalies for stations in France between 1948 and 2007 are shown in Fig. 5. This figure also shows the return levels (RL) of summer TX for a return period of 50 years. This RL is estimated by adding the mean summer TX to the RL of the anomaly. Such values might be more meaningful to policymakers who have to consider actual temperatures rather than anomalies. The shape parameters of temperature are all negative, indicating a short tailed distribution (Parey et al., 2007). The scale parameter $\sigma$ indicates a gradient of increasing temperature variability from the south east to the north west of France. This gradient translates into a gradient in RLs across France. Hence, apart from a couple of Mediterranean stations, return levels of high TX anomalies are higher in north western France than in 

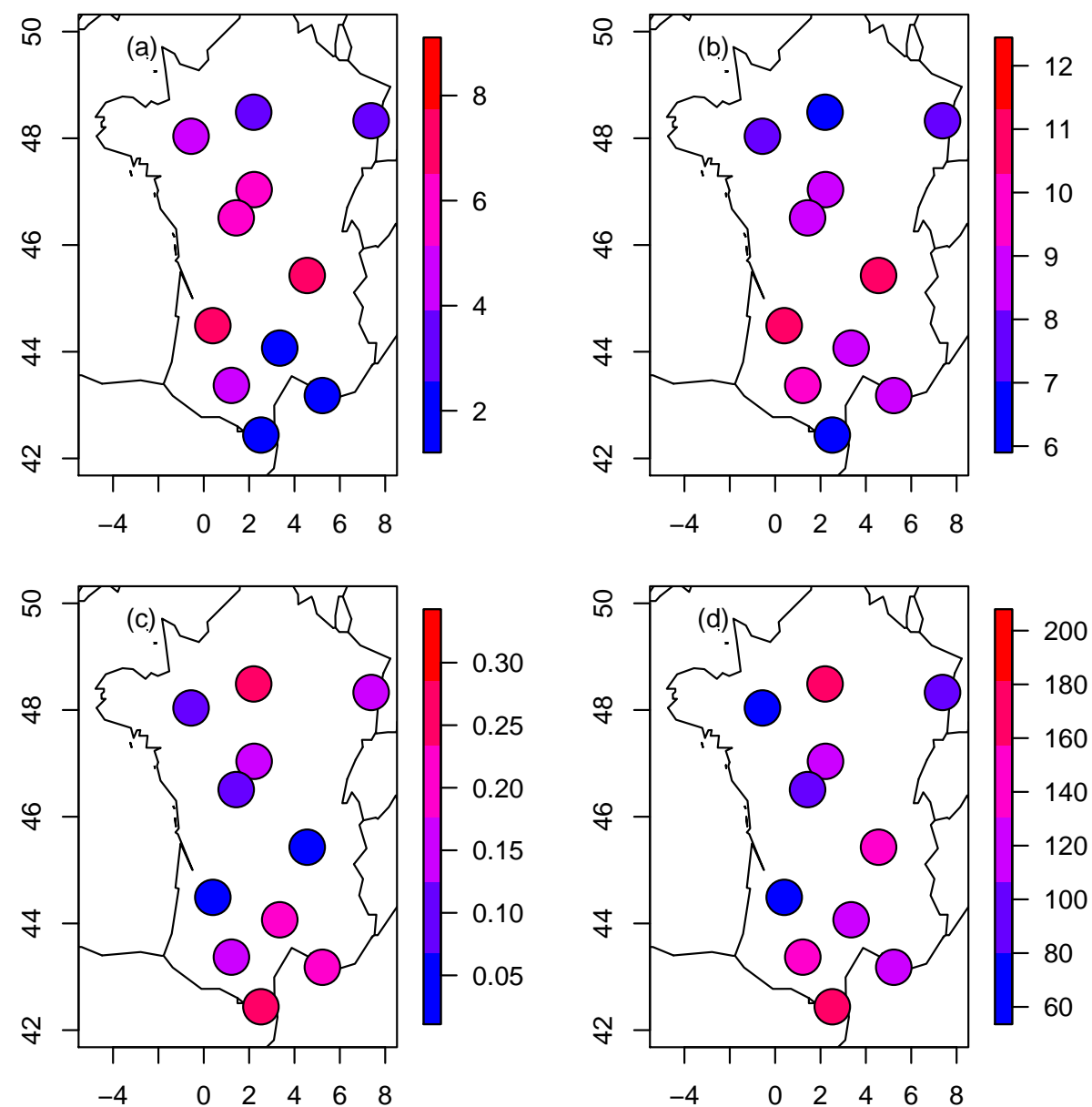

Fig. 6. Generalized Pareto Distribution (GPD) parameters of daily summer precipitation (RR) for the French ECA stations (Klein-Tank et al., 2002) between 1948 and 2007. Panel (a): threshold $u$ in mm/day; (b) scale $\sigma$ in mm/day; (c) shape $\xi$; (d) return level for 50 year period $\mathrm{RL}_{50}$ in $\mathrm{mm} /$ day.

the south east. Generally warm summer temperatures every year in southern France can explain this, implying little interannual variability (and small return levels for TX anomalies). On the other hand, summer temperature variability is higher in western France, with the possibility of heatwaves as well as cold temperatures, implying high return levels for TX anomalies.

The GPD parameters of summer RR for stations in France between 1948 and 2007 are shown in Fig. 6. The shape parameters of precipitation are all positive, indicating a heavy tailed distribution for daily precipitation. Thus, as suspected from observation, summer precipitation yields an important variability for its extremes.

We do not find significant changes in the GPD parameters of TX or RR when considering the whole time series, i.e. starting before 1948 (not shown) and since 1948. Thus, those estimates are stable in time, which comforts our stationary hypothesis.
For further simplification purposes, we chose four stations (Paris, Perpignan, Rennes and Strasbourg) to illustrate the changes in GPD parameters when the EVT analysis is done with weather regime conditioning (Sect. 2.3). The GPD parameters for TX of those stations are shown in Fig. 7. For those stations, the weather regimes yield significant changes in the extreme parameter estimates.

We observe that the return levels associated to weather regimes can be significantly different from the raw ones. For TX data, the shape parameters do not change much (Fig. 7). Thus the RL estimates for TX, although sometimes significant (e.g. Paris, Rennes, Bourges, and Lyon), do not depend systematically on the weather regimes. Over the 27 stations in France, we find that 26 show a regime dependence for the threshold $u, 11$ stations for the scale parameter $\sigma$ and 14 stations for the shape parameter $\xi$ (not shown). This can translate into changes that can be as large as $4^{\circ} \mathrm{C}$ in the threshold $u$, and hence in a return level estimate (every other GPD parameter being equal). We note that the 

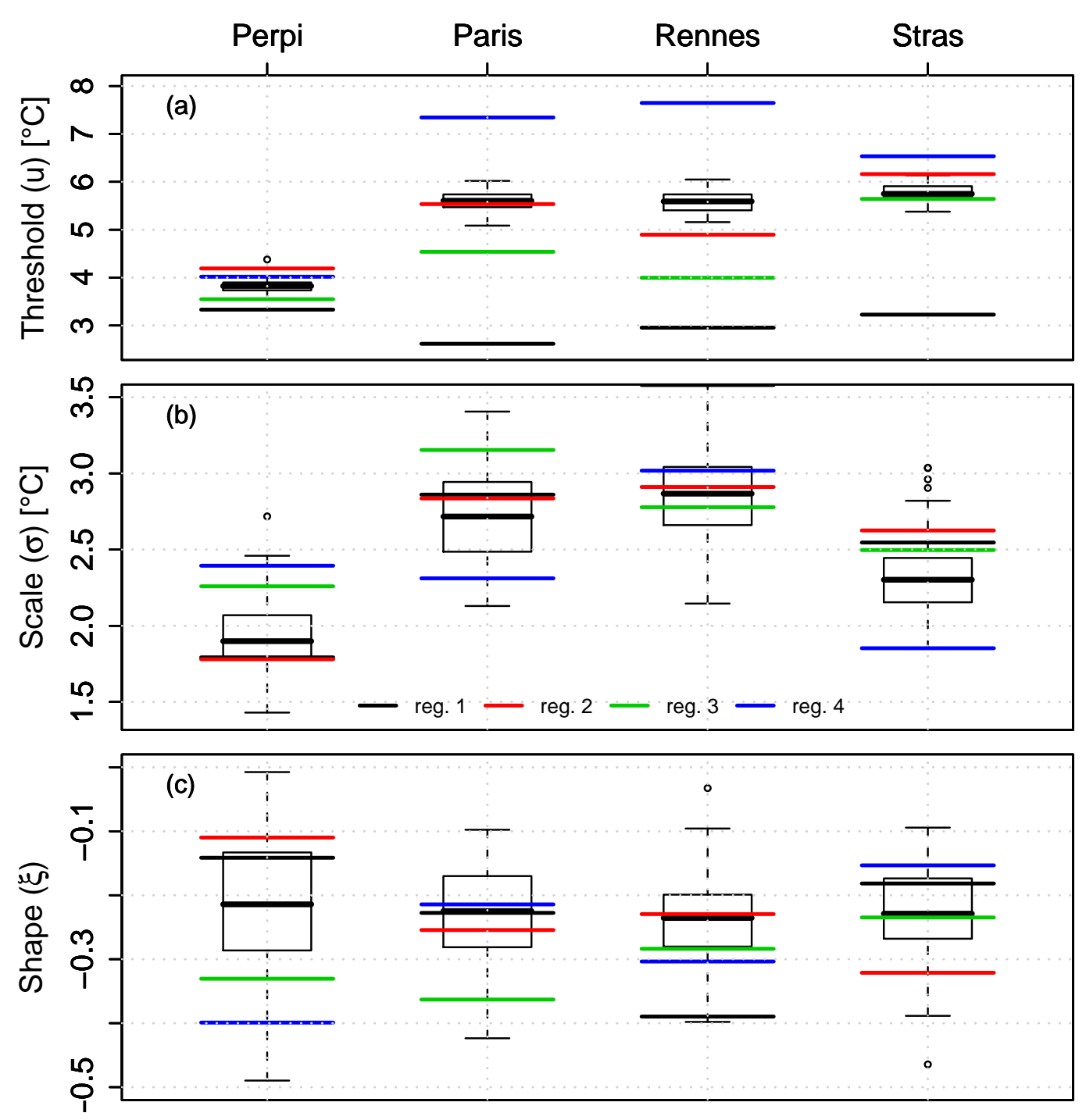

Fig. 7. Weather regime dependence of Generalized Pareto Distribution (GPD) parameters (threshold, scale and shape) of daily maximum temperature (TX) anomalies for four French stations (Perpignan, Paris, Rennes and Strasbourg) from the ECA dataset (Klein-Tank et al., 2002) between 1948 and 2007. Panels (a-c) respectively account for threshold estimates $(u)$, scale $(\sigma)$ and shape $(\xi)$ parameters. Box and whisker plots are obtained by boostrap experiments with randomly distributed weather regimes, as described in Sect. 2.3: the top and bottom horizontal lines represent the 5th and 95th quantiles, and the boxes are defined by the 25th, 50th and 75th quantile. Circles indicate outliers. For comparison purposes, the horizontal colored lines (black, red, green and blue) indicate the GPD estimates conditioned by the four weather regimes.

difference in RLs between weather regime dependent estimates and raw estimates is overall positive for anticyclonic regimes (regimes 2 and 4 ) and negative for cyclonic regimes (regimes 1 and 3), especially for small return periods. In combination with Fig. 4e, this suggests that warm summers on average (with anticyclonic regimes) experience hot extreme temperatures. Conversely, cold summers with cyclonic conditions yield lower temperature extremes. Although this is close to a physical intuition, we note that this relation should be interpreted with care because it obviously depends on ocean proximity and altitude.
The same analysis was performed on precipitation (RR) data. It appears that the shape parameter can switch signs with the regime conditioning with statistical significance (e.g. Paris, see Fig. 8c). Over the 11 stations in France, we also find that 11 show a regime dependence for the threshold $u, 8$ stations for the scale parameter $\sigma$ and 5 stations for the shape parameter $\xi$ (not shown). This has important consequences on the return level estimates which are sensitive to this parameter (Fig. 9). For the Paris data, we note that the heavier precipitations occur during regimes 1 (NAO-) and 4 (BLO). This is not surprising for regime 1 , which is 

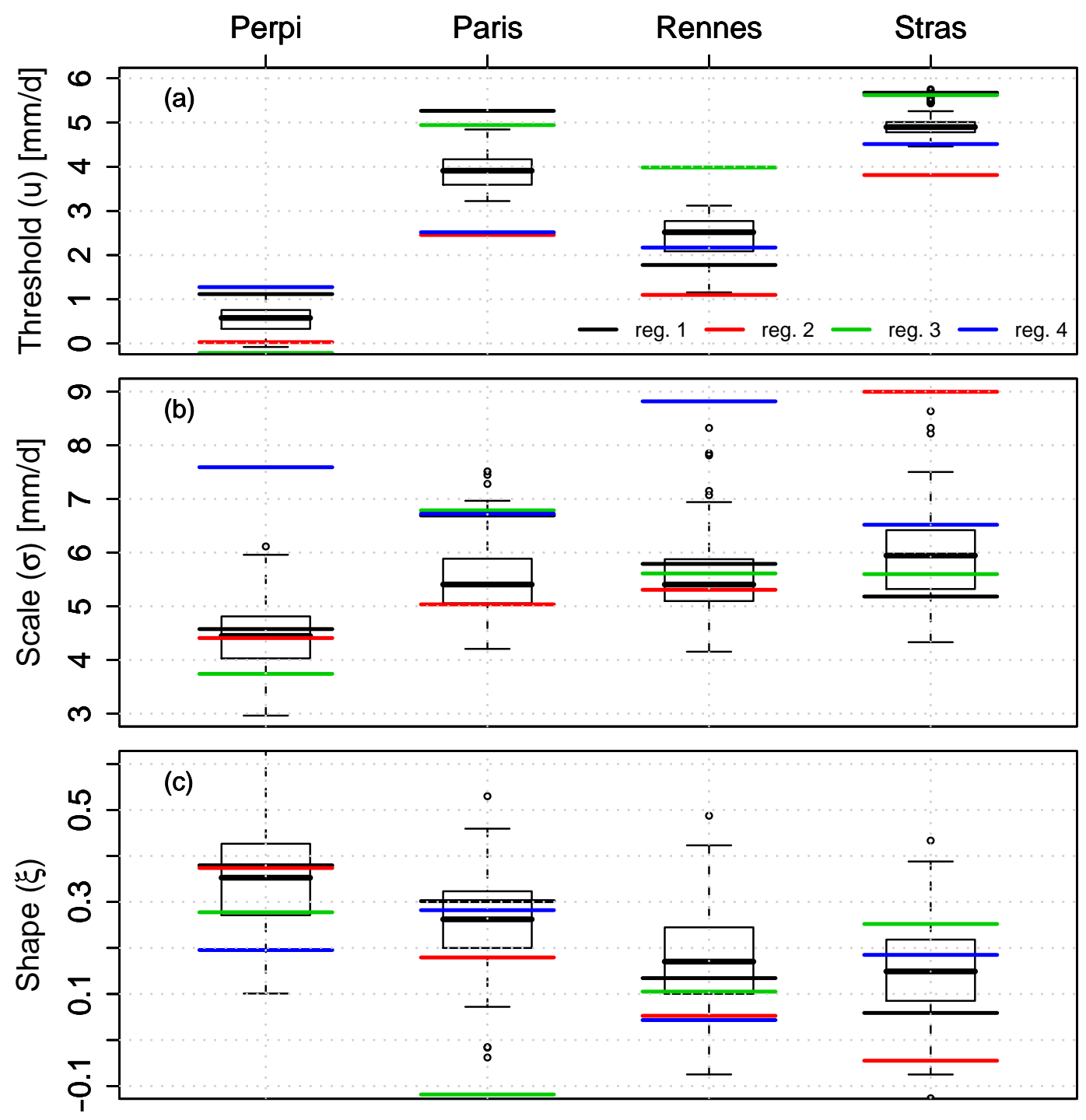

Fig. 8. Weather regime dependence of Generalized Pareto Distribution (GPD) parameters of daily precipitation (RR) for four French stations (Perpignan, Paris, Rennes and Strasbourg) from the ECA dataset (Klein-Tank et al., 2002) between 1948 and 2007. Panels and lines are the same as for Fig. 6.

cyclonic over Western Europe and is associated with most wet summers. But regime 4 is rarer than usual during generally wet summers and is often encountered during European droughts and heatwaves (Cassou et al., 2005; Fischer et al., 2007). Thus this analysis suggests that in Paris (and Lyon), generally dry summers can yield heavy precipitation (e.g. in the form convective episodes) which have daily time scales. Moreover, generally wet summers associated with the cyclonic regime $3(\mathrm{GA})$ do not yield such heavy precipitation. During such years, precipitation is steady (with rather cool temperatures) throughout the summer, but there are no (or few) convective episodes bring intense precipitation. Thus, in a station like Paris, the most intense precipitation can be found during summers dominated by a block- ing regime, which tends to favor overall dry and warm summers, or those with lasting depressions over northern Europe (which is the case for the summer 2007, for example).

\section{Conclusions}

In this paper, we presented a heuristic methodology to model the dependence of climate extremes to a physically relevant variable like the atmospheric circulation, generalizing previous approaches (Plaut et al., 2001; Plaut and Simonnet, 2001; Robertson and Ghil, 1999; Simonnet and Plaut, 2001; Yiou and Nogaj, 2004) by using the extreme value theory. 

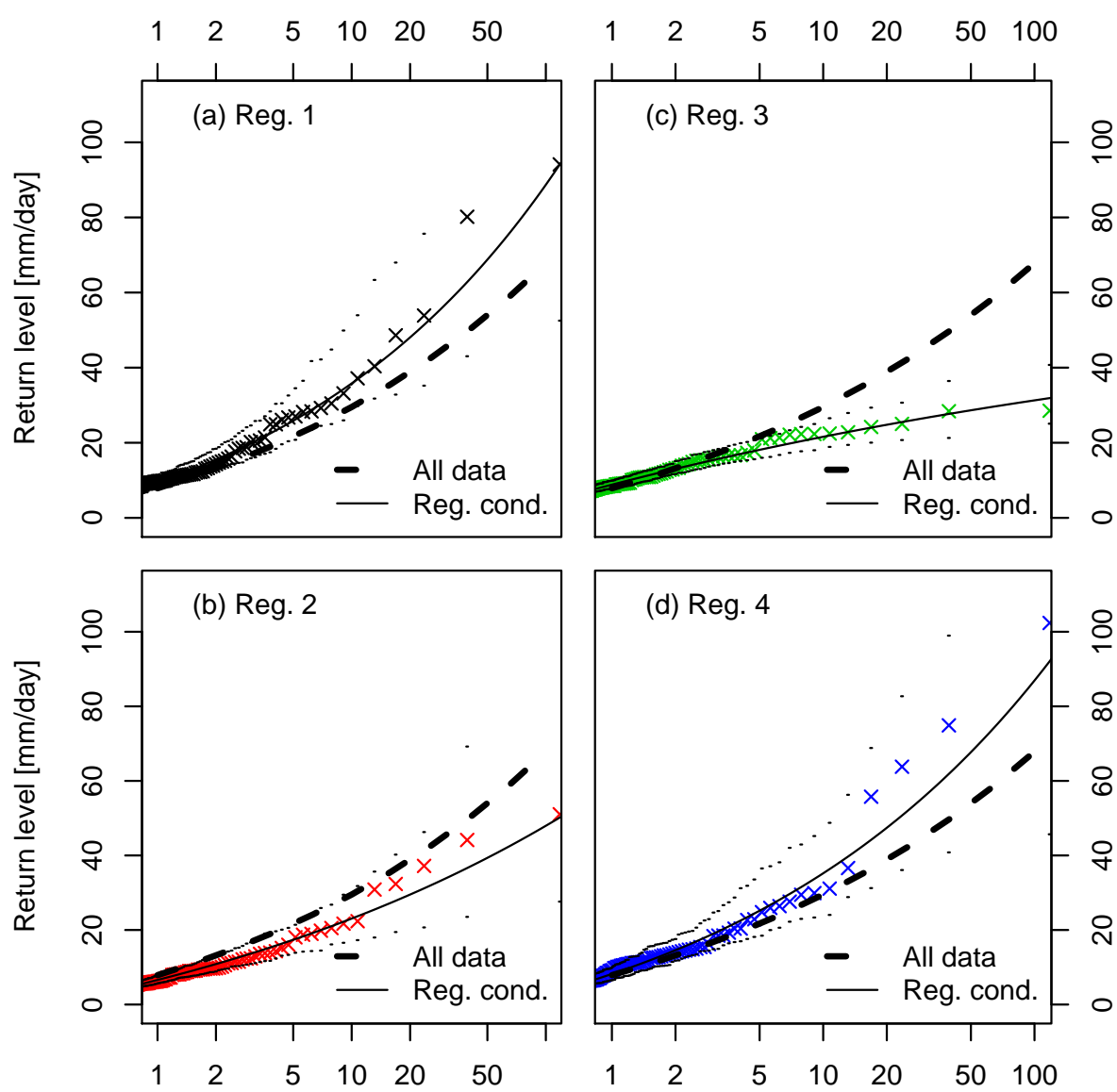

Return period [Yr]

Return period $[\mathrm{Yr}]$

Fig. 9. Return levels (RLs) as functions of return periods, for Paris daily summer precipitation (RR; ECA dataset (Klein-Tank et al., 2002)) between 1948 and 2007, conditioned by the four weather regimes (panels a-d) shown in Fig. 4a-d. The thin continuous lines indicate the weather regime dependent RLs, the dotted lines are the $95 \%$ confidence intervals. The colored crosses $(x)$ represent the data. The thick dashed lines represent the RL of RR without weather regimes (see Fig. 3a).

We focused on observations of daily maximum temperature (TX) and daily precipitation (RR) (Klein-Tank et al., 2002) in France and related their extremes to weather regimes obtained from the daily geopotential height at $500 \mathrm{mb}$ from the NCEP reanalysis (Kalnay et al., 1996).

We find that the hottest summers in Europe (which generate high temperature anomalies over France) are consistent with anticyclonic conditions over Western Europe. Our analysis suggests that the corresponding weather regimes enhance the probability of daily temperature extremes. This weather regime enhancement can be as large as $4^{\circ} \mathrm{C}$ (e.g. in Paris) and this should be taken into account in operational extreme weather alerts. The close relation between the mean behavior of temperature and its extremes can be explained by its bounded tail (with negative shape parameters) which forces the extremes to vary like the center of the distribution (Nogaj et al., 2007). Hence we can claim that hot summers generally bear hot extreme temperatures.
On the other hand, wet European summers are generally associated with cyclonic conditions (bringing moist air from the ocean) over Western Europe. Our results show that, unlike temperature, the behavior of extreme precipitation is strongly affected by weather regime conditioning. In particular, the shape parameter of the GPD distribution can change signs with the weather regime dependence, from heavy tail to short tail distribution. We observe that the most intense extremes for precipitation seem to coincide with anticyclonic conditions (which tend to favor dry summers). We observe that the most intense extreme precipitation coincides with regime 1 (NAO-) related to cyclonic conditions, but also with regime 4 (Blocking), which tends to favor dry summers and is characterized by an anticyclonic pattern. We explain this by the fact that intense convective episodes, bringing flash precipitation, often occur at the end of heatwaves, which bear dry conditions in Europe. Hence, overall dry and hot summers in France can yield episodes of extreme 
precipitation. In contrast, generally wet summers do not necessarily have extreme precipitation and might be due to moderate but steady rains (like the seasons dominated by regime 3 (Greenland Anticyclone)).

Our approach completes the analysis of Vrac and Naveau (2007) who modeled the dependence of precipitation over the US by taking the atmospheric circulation into account. Our study justifies that such modeling can be applied on precipitation and temperature by assessing the statistical significance of this dependence. In this paper, we find some spatial coherence for the extreme parameters of temperature, but we did not consider joint extreme distribution or spatial extremes for which EVT requires further developments (e.g. Vannitsem and Naveau, 2007).

Methodological refinements could be added to this promising but heuristic approach. In particular, the time dependence of the POT is only considered through the sequence of weather regimes, which can be seen as a Markov chain (Ghil and Robertson, 2002). A generalization of the results of Smith et al. (1997) to temperature or precipitation conditioned by a hidden Markov chain could be useful for a thorough model of extreme distributions.

Acknowledgements. We are thankful to the thoughtful suggestions of three anonymous reviewers who helped clarify the manuscript. This work was supported by the EC Project "Extreme Events: Causes and Consequences" (E2-C2), Contract No. 12975. All calculations were done in R (www.r-project.org).

Edited by: B. D. Malamud

Reviewed by: three anonymous referees

\section{References}

Beck, C., Jacobeit, J., and Jones, P.: Frequency and within-type variations of large-scale circulation types and their effects on low-frequency climate variability in Central Europe since 1780, Int. J. Climatol., 27, 473-491, 2007.

Bernacchia, A. and Naveau, P.: Detecting spatial patterns with the cumulant function. Part I: The theory, Nonlin. Processes Geophys., 15, 159-167, 2007, http://www.nonlin-processes-geophys.net/15/159/2007/.

Bradley, R. S.: Paleoclimatology: Reconstructing Climates of the Quaternary, Academic Press, San Diego, 1999.

Cassou, C., Terray, L., and Phillips, A.: Tropical Atlantic influence on European heat waves, J. Climate, 18, 2805-2811, 2005.

Cheng, X. H. and Wallace, J. M.: Cluster analysis of the northernhemisphere wintertime 50-hPa height field: spatial patterns, J. Atmos. Sci., 50, 2674-2696, 1993.

Coles, S.: An Introduction to Statistical Modeling of Extreme Values, Springer Series in Statistics, Springer-Verlag, London, 2001.

Cooley, D., Naveau, P., Jomelli, V., Rabatel, A., and Grancher, D.: A Bayesian hierarchical extreme value model for lichenometry, Environmetrics, 17, 555-574, 2006.

Corti, S., Molteni, F., and Palmer, T. N.: Signature of recent climate change in frequencies of natural atmospheric circulation regimes, Nature, 398, 799-802, 1999.
Efron, B. and Tibshirani, R.: An introduction to the bootstrap, Monographs on statistics and applied probability; 57, Chapman and Hall, New York, 1993.

Embrechts, P., Klüppelberg, C., and Mikosch, T.: Modelling Extremal Events for Insurance and Finance, Applications of mathematics, 33, Springer, Berlin; New York, 1997.

Fischer, E., Seneviratne, S., Luthi, D., and Schaer, C.: Contribution of land-atmosphere coupling to recent European summer heat waves, Geophys. Res. Lett., 34, L05707, doi:10.1029/2006GL029068, 2007.

Ghil, M. and Robertson, A. W.: "Waves" vs. "particles" in the atmosphere's phase space: A pathway to long-range forecasting?, Proc. Natl. Acad. Sci., 99, 2493-2500, 2002.

Green, P. J. and Silverman, B. W.: Nonparametric regression and generalized linear models: a roughness penalty approach, Monographs on statistics and applied probability; 58, Chapman and Hall, London; New York, 1st edn., 1994.

Gumbel, E. J.: Statistics of Extremes, Columbia University Press, New York, 1958.

Hartigan, J. A. and Wong, M. A.: A K-means clustering algorithm., Appl. Statist., 28, 100-108, 1979.

Hastie, T. and Tibshirani, R.: Generalized additive models, Monographs on statistics and applied probability; 43, Chapman and Hall, London, New York, 1st edn., 1990.

Hurrell, J., Kushnir, Y., Ottersen, G., and Visbeck, M. (Eds.): An Overview of the North Atlantic Oscillation. The North Atlantic Oscillation: Climate Significance and Environmental Impact, vol. 134 of Geophysical Monograph Series, American Geophysical Union, 2003.

Kageyama, M., D’Andrea, F., Ramstein, G., Valdes, P. J., and Vautard, R.: Weather regimes in past climate atmospheric general circulation model simulations, Clim. Dynam., 15, 773-793, 1999.

Kalnay, E., Kanamitsu, M., Kistler, R., Collins, W., Deaven, D., Gandin, L., Iredell, M., Saha, S., White, G., Woollen, J., Zhu, Y., Leetmaa, A., Reynolds, R., Chelliah, M., Ebisuzaki, W., W.Higgins, Janowiak, J., Mo, K. C., Ropelewski, C., Wang, J., Jenne, R., and Joseph, D.: The NCEP/NCAR 40-Year Reanalysis Project, B. Am. Meteorol. Soc, 77, 437-471, 1996.

Katz, R., Parlange, M., and Naveau, P.: Statistics of extremes in hydrology, Adv. Water Res., 25, 1287-1304, 2002.

Katz, R. W.: Extreme value theory for precipitation: sensitivity analysis for climate change, Adv. Water Res., 23, 133-139, 1999.

Kharin, V. V. and Zwiers, F. W.: Changes in the extremes in an ensemble of transient climate simulations with a coupled atmosphere-ocean GCM, J. Climate, 13, 3760-3788, 2000.

Kimoto, M. and Ghil, M.: Multiple flow regimes in the Northernhemisphere winter. 1. Methodology and hemispheric regimes, J. Atmos. Sci., 50, 2625-2643, 1993a.

Kimoto, M. and Ghil, M.: Multiple flow regimes in the Northern Hemisphere winter. Part II: Sectorial regimes and preferred transitions, J. Atmos. Sci., 50, 2645-2673, 1993b.

Klein-Tank, A., Wijngaard, J., Konnen, G., Bohm, R., Demaree, G., Gocheva, A., Mileta, M., Pashiardis, S., Hejkrlik, L., KernHansen, C., Heino, R., Bessemoulin, P., Muller-Westermeier, G., Tzanakou, M., Szalai, S., Palsdottir, T., Fitzgerald, D., Rubin, S., Capaldo, M., Maugeri, M., Leitass, A., Bukantis, A., Aberfeld, R., Van Engelen, A., Forland, E., Mietus, M., Coelho, F., Mares, C., Razuvaev, V., Nieplova, E., Cegnar, T., Lopez, J., 
Dahlstrom, B., Moberg, A., Kirchhofer, W., Ceylan, A., Pachaliuk, O., Alexander, L., and Petrovic, P.: Daily dataset of 20thcentury surface air temperature and precipitation series for the European Climate Assessment, Int. J. Climatol., 22, 1441-1453, 2002.

Leadbetter, M. R., Lindgren, G., and Rootzén, H.: Extremes and Related Properties of Random Processes, Springer Series in Statistics, Springer-Verlag, Berlin, 1982.

Luterbacher, J., Schmutz, C., Gyalistras, G., Xoplaki, E., and Wanner, H.: Reconstruction of monthly NAO and EU indices back to AD 1675, Geophys. Res. Lett., 26, 2745-2748, 1999.

Michelangeli, P. A., Vautard, R., and Legras, B.: Weather regimes: Recurrence and quasi stationarity, J. Atmos. Sci., 52, 1237-1256, 1995.

Naveau, P. and Moncrieff, M.: A probabilistic description of convective mass fluxes and its relationship to extreme-value theory, Q. J. Roy. Meteorol. Soc., 129, 2217-2232, 2003.

Naveau, P., Nogaj, M., Ammann, C., Yiou, P., Cooley, D., and Jomelli, V.: Statistical methods for the analysis of climate extremes, C R. Acad. Sci. Geoscience, 337, 1013-1022, 2005.

Nogaj, M., Yiou, P., Parey, S., Malek, F., and Naveau, P.: Amplitude and frequency of temperature extremes over the North Atlantic region, Geophys. Res. Lett., 33, L10801, doi:10.1029/2005GL024251, 2006.

Nogaj, M., Parey, S., and Dacunha-Castelle, D.: Non-stationary extreme models and a climatic application, Nonlin. Processes Geophys., 14, 305-316, 2007, http://www.nonlin-processes-geophys.net/14/305/2007/.

Palmer, T. N.: A nonlinear dynamical perspective on climate prediction, J. Climate, 12, 575-591, 1999.

Parey, S., Malek, F., Laurent, C., and Dacunha-Castelle, D.: Trends and climate evolution: Statistical approach for very high temperatures in France, Clim. Change, 81, 331-352, 2007.

Plaut, G. and Simonnet, E.: Large-scale circulation classification, weather regimes, and local climate over France, the Alps and Western Europe, Clim. Res., 17, 303-324, 2001.

Plaut, G., Schuepbach, E., and Doctor, M.: Heavy precipitation events over a few Alpine sub-regions and the links with largescale circulation, 1971-1995, Clim. Res., 17, 285-302, 2001.

Robertson, A. W. and Ghil, M.: Large-scale weather regimes and local climate over the Western United States, J. Climate, 12, 1796$1813,1999$.
Simonnet, E. and Plaut, G.: Space-time analysis of geopotential height and SLP, intraseasonal oscillations, weather regimes, and local climates over the North Atlantic and Europe, Clim. Res., 17, 325-342, 2001.

Smith, R., Tawn, J., and Coles, S.: Markov chain models for threshold exceedances, Biometrika, 84, 249-268, 1997.

Smyth, P., Ide, K., and Ghil, M.: Multiple regimes in Northern hemisphere height fields via mixture model clustering, J. Atmos. Sci., 56, 3704-3723, 1999.

Vannitsem, S. and Naveau, P.: Spatial dependences among precipitation maxima over Belgium, Nonlin. Processes Geophys., 14, 621-630, 2007, http://www.nonlin-processes-geophys.net/14/621/2007/.

Vautard, R.: Multiple weather regimes over the North Atlantic: Analysis of precursors and successors, Mon. Weather Rev., 118, 2056-2081, 1990.

Vautard, R. and Legras, B.: On the source of midlatitude lowfrequency variability. 2. Nonlinear equilibration of weather regimes, J. Atmos. Sci., 45, 2845-2867, 1988.

Vautard, R., Legras, B., and Déqué, M.: On the source of midlatitude low-frequency variability.1. A statistical approach to persistence, J. Atmos. Sci., 45, 2811-2843, 1988.

Vautard, R., Yiou, P., D'Andrea, F., de Noblet, N., Viovy, N., Cassou, C., Polcher, J., Ciais, P., Kageyama, M., and Fan, Y.: Summertime European heat and drought waves induced by wintertime Mediterranean rainfall deficit, Geophys. Res. Lett., 34, L07711, doi:10.1029/2006GL028001, 2007.

von Storch, H. and Zwiers, F. W.: Statistical Analysis in Climate Research, Cambridge University Press, Cambridge, 2001.

Vrac, M. and Naveau, P.: Stochastic downscaling of precipitation: From dry events to heavy rainfalls, Water Resour. Res., 43, W07402, doi:10.1029/2006WR005308, 2007.

Vrac, M., Hayhoe, K., and Stein, M.: Identification and intermodel comparison of seasonal circulation patterns over North America, Int. J. Climatol., 27, 603-620, 2007.

Yiou, P. and Nogaj, M.: Extreme climatic events and weather regimes over the North Atlantic: When and where?, Geophys. Res. Lett., 31, L07202, doi:10.1029/2003GL019119, 2004.

Yiou, P., Ribereau, P., Naveau, P., Nogaj, M., and Brazdil, R.: Statistical analysis of floods in Bohemia (Czech Republic) since 1825, Hydrol. Sci. J., 51, 930-945, 2006. 